\title{
Nanofotónica. Los grandes avances y retos de un mundo pequeño
}

\section{Nanophotonics. The great advances and challenges of a small world}

\author{
Víctor Coello*
}

\begin{abstract}
A general overview of the development of nanophotonics at a global level is presented. The pioneering developments and the problems they faced are exposed. The current trends are also mentioned in a general way. I also describe what are the main reasons that prevent a completed development and what are the strategies to overcome them. Finally, the current situation of this area in Mexico is shown in a descriptive way.
\end{abstract}

KEYWORDS: nanophotonics, nanotechnology, photonics, plasmonics.

RESUMEN: Se presenta una panorámica general del desarrollo de la nanofotónica a nivel global. Se exponen los desarrollos pioneros y los problemas que los mismos enfrentaron. También se comentan de manera general las tendencias actuales y se describe cuáles son los principales motivos que impiden un desarrollo terminado y cuáles son las estrategias para superarlos. Finalmente, se muestra de manera descriptiva la situación actual de esta área en México.

PALABRAS CLAVE: nanofotónica, nanotecnología, fotónica, plasmónica.

\section{Introducción}

Hoy en día, existe el consenso generalizado de definir la nanofotónica como el estudio de la interacción de la luz con la materia en la escala nanométrica. La importancia de la nanofotónica puede ser medida en términos de una creciente actividad científica en las últimas décadas tanto en la dirección tecnológica como en la de fundamento (Ohtsu et al., 2017). En general, la interacción de la luz con la materia provoca tres bien conocidos fenómenos: reflexión, transmisión y absorción. Mientras que los dos primeros pueden ser modelados mediante la óptica geométrica y/o la teoría clásica ondulatoria, el fenómeno de absorción de la luz requiere, generalmente, de una aproximación más compleja. En una escala macroscópica, la absorción de la luz es responsable, entre otras cosas, del origen del color que percibimos en la naturaleza. En una escala microscópica, es decir, en la escala de los fotones, dicha absorción significa que la energía de un fotón es tomada por otras partículas (núcleos, átomos o

Recibido: 6 de febrero de 2019. Aceptado: 25 de abril de 2019.

* Centro de Investigación Científica y de Educación Superior de Ensenada, Unidad Monterrey, México.

Correo electrónico: vcoello@cicese.mx 
moléculas) lo que provocará transiciones entre sus niveles de energía. Esta energía electrónica absorbida se puede transformar, a través de distintos procesos, en re-emisión de otros fotones, calor, fonón y plasmón. El plasmón es la cuantización de las oscilaciones del plasma, es parte medular de los avances en la comprensión de los fundamentos de la interacción luz-materia en escalas nanométricas. Un caso particular de este fenómeno es el que ocurre en una interfaz formada por un metal y un dieléctrico y es conocido como plasmón polaritón de superficie (PPS), el cual es a menudo llamado simplemente plasmón superficial (PS) (Raether, 1988). Tomando en cuenta que un polaritón es el resultado de acoplar un fotón con una onda de polarización eléctrica o magnética, los PSs son oscilaciones de la densidad de carga de electrones superficiales que pueden existir en una interfaz metal/dieléctrico y son provocadas por acoplamiento de luz, a una cierta frecuencia, en esa geometría. Asociado a ellos, hay un campo electromagnético que se propaga a lo largo de dicha interfaz y que observa decaimientos exponenciales perpendiculares a la misma (ver figura 1). Por esta razón, los PSs exhiben una sensibilidad alta a las propiedades de la superficie incluida su rugosidad.

Para que exista físicamente un PS, el número de onda asociado a este debe ser mayor (en valor absoluto) al número de onda de la luz en la vecindad del medio, lo cual es una característica inherente de los campos evanescentes (Fornel, 2001). Los PSs obedecen a las ecuaciones de Maxwell y representan ondas (cuasi) bidimensionales. La derivación electromagnética de estos modos resulta en el hecho de que son posibles solo para la polarización $p$ de la luz (ondas TM) ya que las ondas con polarización $s$ (TE) no satisfacen las condiciones de frontera para la geometría descrita con anterioridad. Debido a esta naturaleza electromagnética, no es difícil inferir que los PSs pueden difractarse, reflejarse e interferir. Estas propiedades se muestran claramente en el curso de la propagación de ellos. En este mismo fenómeno

FIGURA 1. Representación pictórica de un plasmón polaritón de superficie.

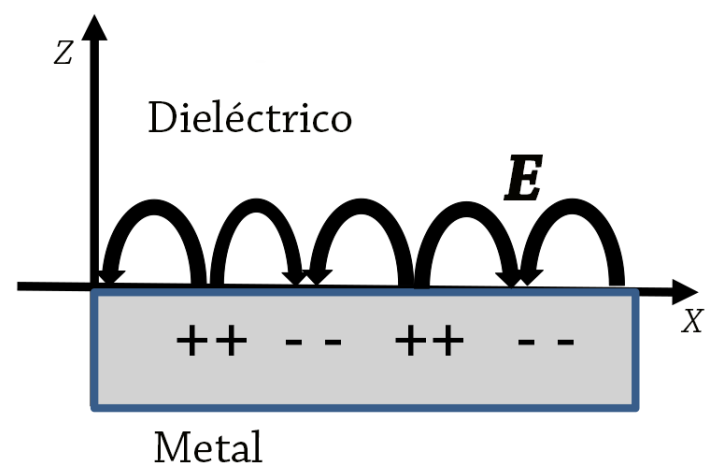

Fuente: Elaboración del autor. 
de propagación superficial, el PS experimenta el llamado fenómeno de esparcimiento (Bozhevolnyi y Coello, 1998). El esparcimiento del PS es causado casi siempre por imperfecciones superficiales dado que incluso las superficies más cuidadosamente preparadas presentan algún tipo de rugosidad relativa. En lo sucesivo, debemos distinguir entre dos tipos de esparcimiento de PSs: el inelástico y el elástico. El esparcimiento elástico se produce cuando el PS es reflejado por imperfecciones de la superficie a lo largo de la misma, es decir, se refleja en otros PSs que conservan la energía total del modo incidente. Esto, por supuesto, es una situación ideal. El esparcimiento inelástico, por su parte, es considerado como aquellas componentes del campo que, habiendo interactuado con imperfecciones superficiales, son esparcidas fuera de la superficie, esto es, son convertidas a ondas viajeras disminuyendo la energía total almacenada en los PSs. En otras palabras, son consideradas pérdidas $\mathrm{y}$, por lo tanto, un efecto no deseado que en muchos casos es considerable.

Acerca de los mecanismos para la excitación de los PSs se han desarrollado ampliamente dos técnicas: la excitación por medio de la luz y la excitación por medio de electrones. La excitación de PSs por electrones está fuera del alcance de este trabajo (se puede encontrar una descripción general en Raether, 1988). En lo que respecta a la excitación de PSs usando luz, esta se puede lograr en una interfaz plana ideal, ya sea utilizando un acoplador de rejilla, rendijas, agujeros, surcos o mediante dispositivos de reflexión interna total atenuada (ATR, por sus siglas en inglés) como las configuraciones de Otto y Kretschmann (Raether, 1988). En este aspecto, las técnicas ATR fueron tradicionalmente las más utilizadas en los estudios de un gran número de fenómenos de PSs (Raether, 1988); incluyen un sistema dieléctrico de metal y aire, en el que un haz de luz incide sobre la superficie metálica bajo un ángulo mayor que el ángulo crítico de la luz. La excitación se produce en la interfaz entre el aire y el metal, y se reconoce como un mínimo en la dependencia angular de la potencia del haz reflejado. Un análisis de espectros angulares de la excitación del PS permite deducir las características de este, cuyo conocimiento es indispensable para cualquier tipo de estudio sobre estos modos. A pesar del éxito en la comprensión de los fenómenos de PSs usando esta técnica, las mediciones del campo electromagnético de los PSs se deducen de una forma indirecta con la limitante que esto implica. Una medición directa de estos modos no es una tarea sencilla, pues los campos evanescentes no almacenan (en promedio) ninguna energía. Este impedimento experimental sufrió un cambio drástico cuando a principios de los años 70, del siglo pasado, una idea propuesta por Synge (1928), se realizó en un experimento (Ash y Nicholls, 1972). El nacimiento de la técnica, llamada en forma general microscopía óptica de campo cercano (SNOM, por sus siglas en inglés), que tiene su base en la detección de campos evanescentes (ver figura 2), tuvo un gran impacto en la actividad de investigación relacionada con PSs (Kawata, 2001). 
FIGURA 2. Esquema de un SNOM para imágenes de campo cercano de intensidad de polaritones de plasmón de superficie. $\mathrm{LS}_{1}$ y $L S_{2}$ son fuentes de láser a $\lambda_{1}$ y $\lambda_{2}$, respectivamente; $S_{1}$ y $S_{2}$ son filtros ópticos. L es una lente; $M$ un espejo.

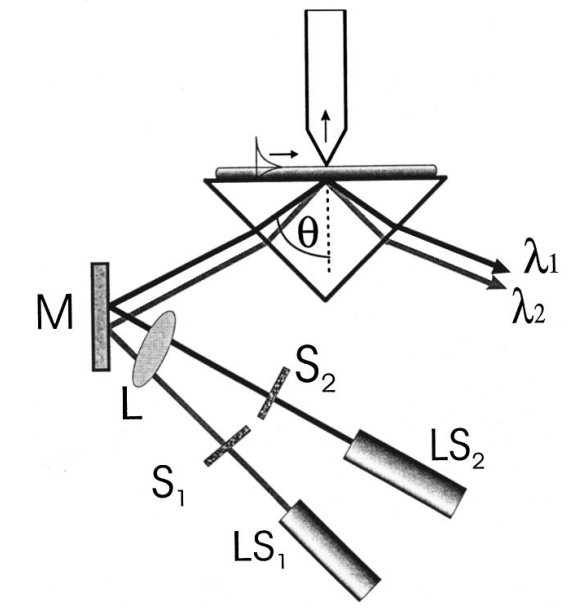

Fuente: Tomado de Bozhevolnyi y Coello (1998).

La posibilidad de una detección directa del PS abrió nuevas direcciones de investigación sobre los fenómenos relacionados con este. La propagación de PSs a lo largo de la superficie que los soporta es uno de los fenómenos más interesantes en este contexto. Como ya se mencionó, el comportamiento de estos modos, incluida su propagación, se ha reconstruido tradicionalmente a partir de medidas angulares de la luz dispersada hacia el espacio libre debido a la interacción de los plasmones con la superficie. Con el nacimiento de las técnicas SNOM, se abrió la posibilidad de estudios locales de la propagación y dispersión de los PSs. En general, uno de los problemas más serios que enfrenta esta forma de detección está relacionado con el hecho de que las distribuciones de intensidad de campo de los PSs son, a menudo, difíciles de interpretar. La relación entre una imagen obtenida mediante SNOM y la distribución de intensidad del modo PS que existe en la ausencia de la sonda de un SNOM (típicamente una punta afilada de fibra óptica) está lejos de ser trivial. El problema se vuelve crítico cuando la punta de la fibra no actúa como un elemento pasivo en la detección de los campos ópticos. Lo anterior puede ser debido a efectos simples como la desalineación del sistema (Merlo et al., 2014) o aún más drásticos como los son el desgaste o daño de la sonda. Mas aún, una punta de fibra homogénea no se puede considerar como un detector pasivo del módulo cuadrado del campo cercano eléctrico (Carminati y Greffet, 1995). En otras palabras, las propiedades de detección de la sonda siempre influyen en el contraste de la imagen obtenida. Dadas estas condiciones, es claro que se debe desarrollar un procedimiento experimental cuidadoso y sistemático para verificar la influencia de la sonda. Como paso siguiente, la modificación del contraste de campo cercano, inducida por la 
sonda, debe ser un factor bien conocido para afirmar que se puede obtener la información sobre las distribuciones de intensidad de un PS que existirían en la ausencia del SNOM. Con el fin de elucidar este problema, se han llevado a cabo gran cantidad de estudios que cubren tanto aspectos experimentales como teóricos durante las últimas dos décadas. Un caso particular fue la introducción de un factor de corrección de contraste (CCF, por sus siglas en inglés). Dicho CCF toma en cuenta el filtraje espacial de frecuencias que se efectúa debido a la sonda de un SNOM al detectar la señal óptica (Coello et al., 1997). El valor del CCF se obtuvo a partir de mediciones de un patrón de interferencia de una onda evanescente estacionaria, generado por dos ondas evanescentes contra-propagantes producidas por la reflexión interna total de la luz dentro de un prisma de vidrio. La influencia del esparcimiento inelástico de los PSs en las distribuciones de intensidad resultantes se exploró en detalle al tomar imágenes ópticas a diferentes distancias de la superficie de la punta para películas lisas y ásperas (oro y plata). Los experimentos confirmaron que las imágenes tomadas cerca de la superficie representan las distribuciones del campo electromagnético, a lo largo de la muestra, siempre y cuando se tome en cuenta el CCF. Tal progreso en la comprensión de las medidas locales de las propiedades del plasmón superficial, estimuló la investigación en muchas direcciones, siendo una de ellas las investigaciones sobre el control local de la propagación de los PSs (Zayats et al., 2005). La similitud de estos modos superficiales y las ondas que se propagan en guías de onda planas evocó la idea fascinante de una óptica integrada de plasmones superficiales, conocida hoy en día como plasmónica (Stockman et al., 2018).

En este artículo se presenta una panorámica general del desarrollo de la nanofotónica en el mundo, con particularidad en el caso de la plasmónica. Se exponen los desarrollos pioneros y los problemas que los mismos enfrentaron. Se introducen las técnicas más emblemáticas para su detección, las cuales están basadas en microscopías alternativas para acceder a escalas nanométricas en los campos ópticos. También se presentan de manera general las tendencias actuales y se describe cuáles son los principales motivos, al momento, que impiden ciertos desarrollos y cuáles son las estrategias para superarlos. Finalmente, se muestra de manera descriptiva la situación actual de esta área en México.

\section{Trabajos pioneros}

Las investigaciones iniciales sobre el control local de modos plasmónicos confirmaron la posibilidad de crear nanocomponentes bidimensionales tales como espejos, divisores de haz, interferómetros, por mencionar algunos ( $F$ cus Issue, Nature Photonics, 2012). Sin embargo, un notable progreso en esta dirección se logró con la extensión del concepto de banda fotónica (PBG, por sus siglas en inglés) hacia el área de la plasmónica (Yoon et al., 2003). El con- 
cepto de PBG está ligado de manera profunda con el de la difracción de Bragg y predice un gran control de la propagación bidimensional de modos plasmónicos. No obstante, a pesar de resultados iniciales alentadores sobre la preservación elástica de energía en nanoestructuras, las pérdidas asociadas a esta en elementos más complicados como las guías de onda bidimensionales, impidieron más progresos en esa dirección. Varias razones estuvieron involucradas en ellos, entre estas el incipiente desarrollo de las técnicas de nanofabricación y el escaso desarrollo de modelos numéricos completos que permitieran el mejor diseño de las estructuras en términos de la optimización de sus funcionalidades. Con la mejora en las técnicas de fabricación y el surgimiento de modelos robustos para el diseño de nanoestructuras, la caracterización de las mismas (a través de técnicas de SNOM) ahora sufría de los largos tiempos de barrido de la técnica para obtener una sola imagen. Un renovado impulso al aspecto fundamental y al de investigación tecnológica del área de la plasmónica vino con el desarrollo de la microscopía de radiación de fuga (LRM, por sus siglas en inglés) (Drezet et al., 2008) (ver figura 3).

La técnica es simple en su concepción y proporciona un rápido mapeo 2D de modos plasmónicos. Lo anterior es una diferencia importante en funcionalidad comparada con las técnicas SNOM. Además, la técnica LRM presenta la posibilidad de acceso simultáneo al espacio directo y al recíproco. Así, la LRM ha estimulado la investigación de nanofotónica en varias direcciones siendo una de estas la interacción y manipulación con nanoestructuras de los modos plasmónicos. En este contexto, se propusieron, con éxito, nanoestructuras plasmónicas más complejas y funcionales (ver figura 4) tales como interferómetros (Ditlbacher et al., 2002; Coello et al., 2004; Cortes y Coello, 2009), prismas (Radko et al., 2008a), lentes (Radko et al., 2008b), espejos parabólicos (Smolyanivov et al., 2005) y (sobre todo) guías de onda (Fang et al., 2015).

En general, la mayoría de estos trabajos están relacionados con la propagación de PSs a través de elementos periódicos o nanoestructurados; sin embargo, parte del esfuerzo se ha focalizado también en la interacción de PSs con arreglos

FIGURA 3. Esquemático de un LRM. BS, divisor de haz. Lámpara. 01 y 02 , objetivos. NDF, filtro de densidad neutral. BB, filtraje espacial en espacio de Fourier, $\Phi$, ángulo azimutal de referencia. CCD, cámara. PC, computadora.

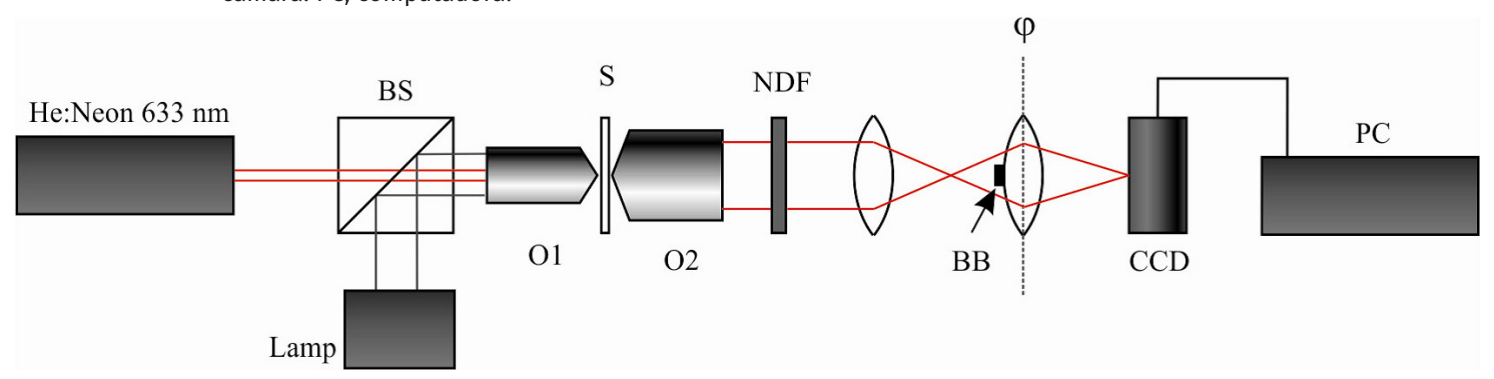

Fuente: Tomado de Coello et al. (2013). 
FIGURA 4. Distintos elementos plasmónicos. A. Espejo parabólico. B. Espejo de Bragg. C. Interferómetro.

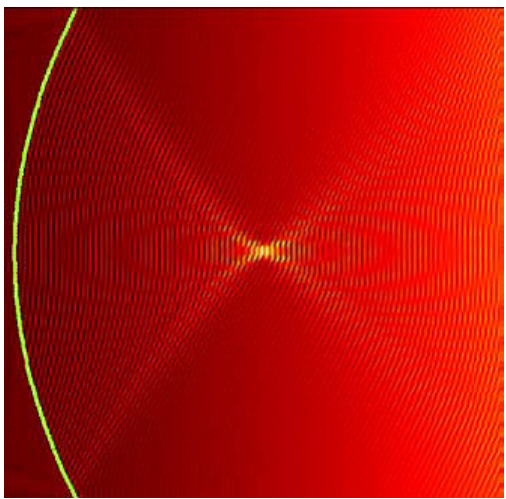

A

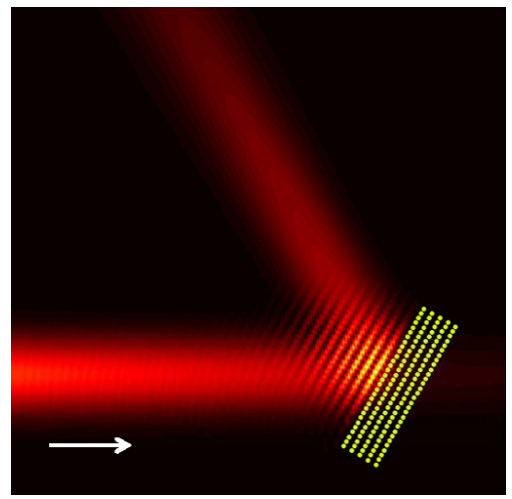

B

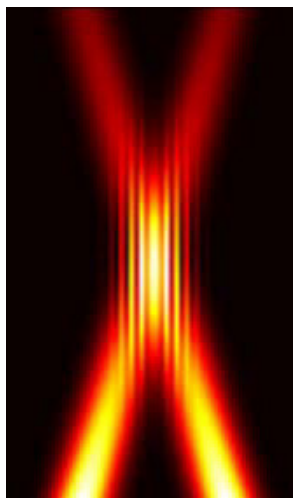

C

Fuente: Tomado de Cortes, y Coello (2009).

de nanopartículas distribuidas al azar (Coello et al., 2013). Los efectos de múltiple esparcimiento, que resultan de dicha interacción, son responsables de la formación de los complicados patrones de interferencia típicos del campo cercano. Este tipo de nociones de fundamento necesariamente necesitan ser entendidas en vías de una optimización de los campos ópticos que son generados, en general, en cualquier nanoestructura plasmónica. De particular interés, es el estudio de los mecanismos físicos que producen el efecto de localización fuerte (tipo Anderson) de los plasmones polaritones de superficie (Maradudin et al., 2001) y hoy en día se mantiene como uno de los estudios más apasionantes relacionados con el área de la plasmónica. Algunos ejemplos de sus aplicaciones prácticas son la posibilidad de una alta densidad de almacenamiento óptico de datos, la mejora del contraste en la espectroscopía local y la generación del segundo armónico (SH) (Coello, 2008). En términos generales, la localización de la luz es un fenómeno enteramente originado debido al esparcimiento elástico múltiple y coherente y su consecuente interferencia en un medio aleatorio. El fenómeno de localización se producirá si la ruta libre media (del PS) es más pequeña o en el orden de $\lambda / 2 \pi$, donde $\lambda$ representa la longitud de onda de la luz. Así, el hecho de que los PSs representen ondas en dos dimensiones permite que puedan ser usados para observar los fenómenos de localización (paralelos a la superficie de metal) como consecuencia del fuerte esparcimiento elástico que sufren en una superficie con una rugosidad relativamente grande. Un caso particular de estudio es la inhibición de la propagación del PS en regiones de superficie con nanopartículas distribuidas espacialmente al azar y el guiado de estos modos en regiones libres de estas partículas (Pisano et al, 2018). El efecto es similar al de PBG y se ha caracterizado usando LRM logrando estructuras clásicas como guías de onda, divisores de haz y mucho más complejas como un espectropolarímetro bidimensional (Chen et al., 2018). 


\section{Tendencias actuales}

A pesar de este desarrollo sin precedentes en nanoestructuras ópticas, siguen existiendo problemas mayores de ciencia básica que si no son resueltos impedirán cualquier oportunidad de explotar las ventajas que las nanoestructuras plasmónicas tienen por ofrecer. Por ejemplo, la interacción de la luz-materia que genera el confinamiento del campo plasmónico en la superficie del metal también induce la concentración del mismo campo dentro del metal. Este mecanismo de absorción significa que los plasmón polaritón de superficie (PPS) tienen una pérdida asociada, la cual en muchos casos es muy considerable. En este sentido, en una interfaz metal-dieléctrico, una disminución de la potencia que es absorbida por el metal se logra aumentando la porción espacial del PPS que se extiende hacia el dieléctrico. No obstante, esto debilita el confinamiento superficial del PPS, lo cual es un efecto no deseado. Una situación inversa existe al usarse mecanismos para aumentar el mencionado confinamiento. Es decir, existe un (inevitable) sacrificio de una cualidad por la otra (conocido en inglés como tradeoff). Sumado a esto, los metales nobles no son compatibles con plataformas basadas en semiconductores complementarios de óxido metálico (CMOS, por sus siglas en inglés) y sus propiedades no se pueden sintonizar o ser ajustadas. Encaminado hacia ello, la comunidad de la nano-óptica junto con la de química sintética y la ingeniería de nanofabricación (entre otras) han hecho esfuerzos para abordar el reto de desarrollar materiales plasmónicos alternativos con propiedades ópticas mejoradas, una fabricación más sencilla y mejores capacidades de integración respecto a los materiales tradicionales (Boltasseva et al., 2011). Los cerámicos, tales como el nitruro de titanio ( $\mathrm{TiN}$ ) y el nitruro de zirconio (ZrN), se encuentran entre los mejores candidatos para sustituir los metales plasmónicos tradicionales. El TiN es compatible con CMOS y proporciona un mayor confinamiento modal en comparación con el oro. Dicho nitruro es muy estable química y térmicamente, biocompatible y extremadamente duro (uno de los cerámicos más duros). El TiN se puede crecer epitaxialmente en muchos sustratos incluyendo zafiro de plano c (c-Al2O3) y silicio [100] formando capas ultra- lisas y ultra- delgadas. Otras de las direcciones en las que se trabaja intensamente es en usar medios activos para compensar la pérdida óhmica a través de la amplificación óptica. Tradicionalmente, la amplificación óptica se ha conseguido con el uso de moléculas de colorante (Seidel et al, 2005), iones de erbio (Ambati et al, 2008) y los puntos cuánticos (QDs) (Bolger et al., 2010). Así, se han reportado compensaciones de pérdida de $~ 30 \%$ para longitudes de onda en el visible, el cercano-infrarojo y telecomunicaciones. Asimismo, es muy difícil obtener compensación de pérdidas por encima del $30 \%$, debido al daño térmico de las estructuras y al fotoblanqueo del medio activo. Por lo tanto, una búsqueda de nuevos y más prometedores medios activos para amplificación de los modos plasmónicos sigue siendo un problema abierto. En este contexto, los nanodiamantes (NDs) 
con centros de vacante de nitrógeno (NV) han demostrado ser buenos candidatos para ser usados como fuentes locales (altamente confinadas) de PS (Kumar et al., 2013) (ver figura 5). La alta intensidad de su fluorescencia y fotoestabilidad permite un uso sistemático en, por ejemplo, guías de ondas plasmónicas donde se necesitan fuentes de PS, reproducibles y estables. Por otra parte, los NDs son excelentes candidatos para ser usados como arreglos desordenados de nanopartículas y de esa forma extender significativamente el entendimiento en los fenómenos inherentes. Por otro lado, un factor clave en la búsqueda de un mejor funcionamiento de las nanoestructuras plasmónicas es el elemento que pueda convertir, eficientemente, la luz hacia PPS. Los elementos tradicionales como antenas, prismas o rejillas resultan ser muy voluminosos para ser integrados en la nanoescala. Con el desarrollo de la nanotecnología, las estructuras de sublongitud de onda en las superficies metálicas, tales como ranuras o crestas, son actualmente investigadas en lo relativo a su eficiencia de acoplamiento luz-PPS. Aquí, uno debe tener en cuenta que, para fines de alta integración, el objetivo es desarrollar un elemento acoplador y un sistema de iluminación que, en conjunto, sean relativamente simples. De acuerdo con lo que ha sido reportado (Radko et al., 2008) y aunque es difícil extender las conclusiones a cualquier sistema sin una investigación previa y exhaustiva, se deduce que una excitación eficiente se puede lograr con un haz de luz enfocado moderadamente y con solo unas pocas crestas o inclusive con una sola. Sin embargo, básicamente solo la incidencia normal de la luz de excitación ha sido considerada en la investigación experimental y muy poca atención se ha puesto a la incidencia oblicua. Se ha predicho en estudios teóricos y comprobado experimentalmente que la incidencia altamente oblicua puede permitir una mayor eficiencia en la generación de PS (Liu et al., 2019).

FIGURA 5. Nanodiamante como posible fuente de fotones individuales.

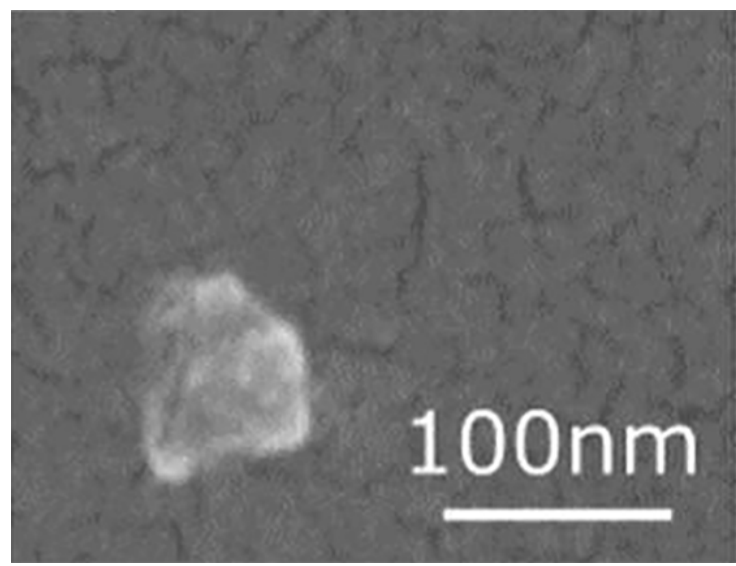

Fuente: Tomado de García, C. et al. (2013). 
Regresando al tema de guiado de PSs, uno de los mayores problemas de las guías de onda plasmónicas está asociado con el débil confinamiento del campo electromagnético en la sección transversal de dichas guías. Y, todavía, hay muchas áreas de oportunidades para la investigación en esos desarrollos. Lo anterior motivado, principalmente, por el gran avance en el desarrollo tecnológico de las técnicas de litografía. Una de estas técnicas, y quizás la más emblemática, es la litografía por haz de electrones (EBL, por sus siglas en inglés). El proceso de EBL (Groves, 2014) es el siguiente: primero, una película metálica de espesor óptico delgado (típicamente de oro) es depositada sobre un sustrato de sílice. En segundo lugar, la muestra es recubierta por medio de centrifugación con polimetilmetacrilato (PMMA), que actúa como una resistencia positiva. Luego, la muestra se graba o estampa utilizando el módulo EBL para su posterior revelado. En este punto, una segunda evaporación se realiza para generar las nanoestructuras metálicas. Finalmente, un solvente (generalmente acetona) se utiliza para disolver la resistencia restante y completar su despegue. Un desarrollo alternativo para las guías de ondas plasmónicas basadas en técnicas de EBL son las llamadas guías de onda cargadas con dieléctrico (DLSPPWs, por sus siglas en inglés). Las DLSPPWs (Holmgaard et al., 2009) han demostrado ser una alternativa atractiva para guiado pues aseguran una buena compensación entre el confinamiento espacial del modo y su distancia de propagación. Varias configuraciones de DLSPPWs han sido demostradas hasta el momento. Entre ellas, uno puede encontrar interferómetros, divisores, y resonadores de anillo de guía de onda (WRRs, por sus siglas en inglés). Un WRR es un bloque de construcción que es factor clave en el desarrollo de circuitos electrónicos-fotónicos integrados. Mediante el uso de EBL se han fabricado WRRs que operan en el infrarrojo cercano. Hay que notar que en el infrarrojo cercano las propiedades plasmónicas tales como longitud de propagación, confinamiento modal y la dispersión del índice efectivo, se espera sean significativamente diferentes en comparación con las de la región visible. Los espectros de transmisión de dichos WRRs fueron experimentalmente medidos y se compararon con expresiones analíticas calculadas. Las estructuras fabricadas mostraron un alto índice de extinción $(\sim 20 \mathrm{~dB})$ y un buen acuerdo entre valores de transmisión calculados y medidos.

Como hemos visto, una de las propiedades más atractivas de los PSs es que se pueden confinar y guiar en nanoestructuras. En general, el funcionamiento y la eficiencia de una guía de onda plasmónica sigue siendo, hoy en día, un desafío. Una buena alternativa a tal problema, es la generación de haces plasmónicos adifraccionales (García et al., 2013). Los haces adifraccionales se caracterizan por tener un perfil de intensidad transversal invariable a lo largo de la dirección de propagación y, además, tienen la capacidad de autorreconstruirse si se coloca un obstáculo a lo largo del eje de propagación. Adicionalmente, la cintura del haz puede ser bastante pequeña, es decir, del orden de una longitud de onda. Tal haz ideal no se puede realizar experimentalmente, al requerir energía y extensión espacial infinita. Sin embargo, 
pueden generarse haces libres de difracción en una región limitada espacialmente. Así, se han propuesto y demostrado de forma experimental la generación de haces plasmónicos adifraccionales usando nanoestructuras fabricadas por medio de EBL, que imitan la funcionalidad de un axicón óptico. En analogía con estos, fue posible generar haces plasmónicos con perfiles de función Bessel de orden cero dentro de una región espacial limitada. Además, una variación de estas nanoestructuras permitió la generación de haces adifraccionales con una gran similitud con funciones Bessel de orden superior. Adicionalmente, los haces plasmónicos adifraccionales generados mostraron la capacidad de autorreconstrucción, siendo esta una de las principales características para haces adifraccionales de tipo Bessel.

Sin lugar a duda, existen muchas variedades de estudios sobre plasmónica adicionales a los presentados en este artículo y que por cuestiones lógicas de espacio no es posible enumerarlos o describirlos en detalle.

El avance en cuestiones de fundamento y de aplicaciones en nanopartículas es un ejemplo de ello. Una extensa revisión y una visión general de las técnicas utilizadas para modelar, fabricar y medir nanopartículas metálicas plasmónicas se puede encontrar en Pelton y Bryant (2013). Las propiedades de las nanopartículas metálicas plasmónicas pueden abarcar aspectos tan interesantes como diversos en relación con su respuesta óptica no lineal de las nanopartículas metálicas y los fenómenos que surgen cuando los materiales emisores de luz se acoplan a las nanopartículas metálicas.

\section{Actualidad en México y conclusiones}

Hoy en día más de 5 mil artículos sobre el área de plasmónica son publicados anualmente. Lo anterior da una idea de la cantidad de preguntas fundamentales que siguen abiertas y que nuevos conceptos científicos y tecnológicos siguen apareciendo. No es posible enumerar la gran lista de desarrollos en un solo artículo, pero, ciertamente, alguna de las tendencias actuales como la supertransmisión, celdas solares, el coloreado plasmónico (sin usar pigmentos) así como la plasmónica cuántica ocupan grandes espacios de desarrollo en este campo (Stockman et al., 2018).

En México se cuenta hoy en día con un desarrollo sólido en este tema. La aparición de grupos de investigación que combinan experiencia y nuevas ideas está creciendo. Se tiene presencia en los cuatro puntos cardinales del país, y en todos y cada uno de los desarrollos descritos en este manuscrito han figurado en mayor o menor alcance. El año internacional de la luz en México (2015) abrió espacios para este tema y reforzó su presencia en el país. La tendencia global de realizar investigaciones multidisciplinarias y de largo aliento también ha fomentado los desarrollos en conjunto de la nanofotónica con la biomedicina. Se enfrenta, sin embargo, un gran reto en materia de desarrollo tecnológico en esta área. El contar, in situ, con un solo espacio que pueda conjuntar los tres aspectos tecnológicos y fundamentales de la nanofotónica: diseño, fabrica- 
ción y caracterización, aún se encuentra en una etapa muy temprana de crecimiento. Lo anterior, por otro lado, abre grandes oportunidades para el desarrollo de proyectos en conjunto. Los avances en estas áreas en las regiones centro, noreste, bajío y norte del país han propiciado ya reuniones interdisciplinarias con miras a buscar objetivos comunes incluido el desarrollo de posgrados en esta rama en específico. En el país, los experimentos y desarrollos numéricos realizados para la comprensión de la nanofotónica se llevan a cabo diariamente. Las investigaciones experimentales y teóricas actuales se consideran como la base para la miniaturización de circuitos fotónicos con escalas de longitud mucho más pequeñas que las que se pueden lograr actualmente. Las aplicaciones se encuentran en sistemas informáticos y sistemas biosensores. El país no debe desaprovechar esta tendencia científico y tecnológica que se da a escala mundial. Las tendencias en nanociencias y en específico en nanofotónica deben ser tomadas como una gran oportunidad para el futuro desarrollo nacional.

\section{Referencias}

Ambati., M., Nam, S., Ulin, E., Genov, D., Bartal, G., Zhang, X. (2008). Observation of stimulated emission of surface plasmon polaritons. Nano Lett., 8: 39984001. http://dx.doi.org/10.1021/nl802603r

Ash, E. y Nicholls, G. (1972). Super-resolution aperture scanning microscope. Nature, 237(5357): 510-512. http://dx.doi.org/10.1038/237510a0

Bolger, P., Dickson, W., Krasavin, A., Liebscher, L., Hickey, S., Skryabin, D., y Zayats, A. (2010). Amplified spontaneous emission of surface plasmon polaritons and limitations on the increase of their propagation length. Opt. Lett., 35: 11971199. http://dx.doi.org/10.1364/OL.35.001197

Boltasseva, A., Atwater, H. (2011). Low-loss plasmonic metamaterials. Science, 331(6015): 290-291. http://dx.doi.org/10.1126/science.1198258

Bozhevolnyi S. I. y Coello V. (1998). Elastic scattering of surface plasmon polaritons: Modeling and experiment. Phys. Rev. B, 58(16): 10899-10910. http://dx.doi.org/10.1103/PhysRevB.58.10899

Carminati, R. y Greffet. (1995). Two-dimensional numerical simulation of the photon scanning tunneling microscope. Concept of transfer function. J. Opt. Commun., 116(4): 316-321. http://dx.doi.org/10.1016/0030-4018(95)91252-W

Coello, V., Bozhevolnyi, S. y Pudonin, F. (1997). Imaging of surface plasmon polaritons with a near-field microscope. Proc. SPIE, 3098: 536-543.

Coello, V., Cortes, R., Garcia, C. y Elizondo, N. (2013). Surface plasmon excitation and manipulation in disordered two-dimensional nanoparticle arrays. NANO: Brief reports and reviews, 8(4): 1350044-1350055.

Coello, V. (2008). Surface plasmon polariton localization. Surface Review Letters, 15(6), 867-879. http://dx.doi.org/10.1142/S0218625X08011974

Coello, V. T. Søndergaard, S. I. Bozhevolnyi. (2004). Modeling of a surface plasmon polariton interferometer. Opts. Comm., 240: 345-350.

http://dx.doi.org/10.1016/j.optcom.2004.06.042 
Cortes, R., y Coello, V. (2009). Modeling of plasmonic phenomena in nanostructured surfaces. NANO: Brief Reports and Reviews, 4(4): 201-216.

Chen, Y., Ding, F., Coello, V. y Bozhevolnyi, S. (2018). On-chip spectropolarimetry by fingerprinting with random surface arrays of nanoparticles. ACS Photonics, 5 (5): 1703-1710. http://dx.doi.org/10.1021/acsphotonics.7b01059

Ditlbacher. H., Krenn, J., Leitner, A., Aussenegg, F. (2002). Two-dimensional optics with surface plasmon polaritons. Applied Physics Letters, 81(10): 1762-1764. http://dx.doi.org/10.1063/1.1506018

Drezet, A., Hohenau, A., Koller, D., Stepanov, A., Ditlbacher, H., Steinberger, B., Aussenegg, F., Leitner, A., Krenn. J. (2008). Leakage radiation microscopy of surface plasmon polaritons. Materials Science and Engineering B, 149(1): 220-229. http://dx.doi.org/10.1016/j.mseb.2007.10.010

Fang, Y. y Sun, M. (2015). Nanoplasmonic waveguides: towards applications in integrated nanophotonic circuits. Light: Science \& Applications, 4: e294. http://dx.doi.org/10.1038/lsa.2015.67

Focus issue, Nature Photonics. (2012). 6(11): 707-794.

Fornel, F. (2001). Evanescent waves from newtonian optics to atomic optics. Alemania: Springer International Publishing.

Holmgaard, T., Z. Chen, S. I. Bozhevolnyi, L. Markey, A. Dereux. (2009). Dielectricloaded plasmonic waveguide-ring resonators. Optics Express, 17(4): 29692975. http://dx.doi.org/10.1364/OE.17.002968

Garcia, C., Coello, V., Han, Z. y Bozhevolnyi, S. (2013). Generation of diffraction-free plasmonic beams with one-dimensional Bessel profiles. Optics Letters, 38(6): 905-907. http://dx.doi.org/10.1364/OL.38.000905

Groves, T. (2014). Electron beam lithography. Nanolithography, Science Direct. http://dx.doi.org/10.1533/9780857098757.80

Kawata, S. (ed.) (2001). Near field optics and surface plasmon polaritons. Alemania: Springer-Verlag, Topics in Applied Physics.

Kumar, S., Huck, A. y Andersen, U. (2013). Coupling of single quantum emitters to plasmons propagating on mechanically etched wires. Opt. Lett., 38: (19) 38383841. http://dx.doi.org/10.1364/OL.38.003838

Liu, H., Lalanne, P., Yang, X. y Hugonin, J. (2008). Surface plasmon generation by subwavelength isolated objects. IEEE Journal of Selected Topics in Quantum Electronics, 14: 1522-1529.

http://dx.doi.org/10.1109/JSTQE.2008.923291

Maradudin, A., Simosen, I., Leskova, T., Mendez, E. (2001). Localization of surface plasmon polaritons on a random surface. Physica B: Condensed Matter., 296(1): 85-97. http://dx.doi.org/10.1016/S0921-4526(00)00784-5

Merlo, J., Coello, V., Cortes, R., Aguilar, F., Flores, A. (2014). Influence of the probesample interaction angle on image formation in apertureless scanning near field optical microscope. Modern Physics Letters B., 28(26): 1450205-1450214. http://dx.doi.org/10.1142/S0217984914502054

Ohtsu, Motoichi, Yatsui, Takashi (eds.) (2017). Progress in nanophotonics 4. Suiza: Springer International Publishing. 
Pelton, M. y Bryant, G. (2013). Introduction to metal-nanoparticle plasmonics. WileyScience Wise Co-Publication.

Pisano, E., Garcia, C., Armenta, F., Garcia, M., Coello, V. (2018). Efficient and directional excitation of surface plasmon polaritons by oblique incidence on metallic ridges. Plasmonics, 13(6): 1935-1940. http://dx.doi.org/10.1007/s11468-018-0708-4

Radko, I., Evlyukhin, A. Boltasseva, A. y Bozhevolnyi, S. (2008a). Refracting surface plasmon polaritons with nanoparticle arrays. Opt. Express, 16(6): 3924-3930. http://dx.doi.org/10.1364/OE.16.003924

Radko, I., Bozhevolnyi, S., Brucoli, G., Martín-Moreno, L., García-Vidal, J., Boltasseva, A. (2008b). Efficiency of local surface plasmon polariton excitation on ridges. Physical Review B, 78:115115-115122. http://dx.doi.org/10.1103/PhysRevB.78.115115

Raether, H. (1988). Surface plasmons. On smooth and rough surfaces and on gratings. Springer Tracts in Modern Physics, 111. Alemania: Springer-Verlag.

Seidel, J., Grafström, S., Eng., L. (2005). Stimulated emission of surface plasmons at the interface between a silver film and an optically pumped dye solution. Phys. Rev. Lett., 94: 177401-177405. http://dx.doi.org/10.1103/PhysRevLett.94.177401

Smolyaninov, I., Davis, C. y Zayats, A. (2005). Image formation in surface plasmon polariton mirrors: applications in high-resolution optical microscopy. New J. Phys., 7: 175-182.

Stockman, M. et al. (2018). Roadmap on plasmonics. J. Opt., 20: 1-39. http://dx.doi.org/10.1088/2040-8986/aaa114

Synge, E. H. (1928). A suggested method for extending the microscopic resolution into the ultramicroscopic region. Phil. Mag., 6(35): 356-362. http://dx.doi.org/10.1080/14786440808564615

Yoon, J., Lee, G., Ho Song, S., Oh, Ch-H. Kim, P. (2003). Surface-plasmon photonic band gaps in dielectric gratings on a flat metal surface. Journal of Applied Physics, 94(1): 123-127. http://dx.doi.org/10.1063/1.1577396

Zayats, A., Smolyaninov, I. y Maradudin, A. (2005). Nano-optics of surface plasmon polaritons. Physics Reports, 408: 131-314.

http://dx.doi.org/10.1016/j.physrep.2004.11.001 